\title{
Controle Ótimo em Epidemias de Dengue
}

\author{
A. MOLTER ${ }^{1 *}$, L.R. PIOVESAN ${ }^{2}$, R. PERGHER ${ }^{1}$ e M.C. VARRIALE ${ }^{3}$
}

Recebido em 20 agosto, 2013 / Aceito em 01 abril, 2016

\begin{abstract}
RESUMO. A infecção pelo vírus da dengue constitui atualmente um dos maiores problemas da saúde pública pelo mundo inteiro. Como ainda não existem vacinas contra os vírus da dengue, é importante ter modelos matemáticos que representem a dinâmica da epidemia visando o controle da propagação da doença. Neste trabalho, utilizamos um sistema SEIR contínuo que modela a dinâmica da transmissão da dengue e fizemos um estudo da estabilidade do modelo com os pontos de equilíbrio biologicamente viáveis. O objetivo deste trabalho é aplicar estratégias de controle alternativas para diminuir a propagação desta doença infecciosa. A estratégia utilizada foi o controle ótimo linear para sistemas não-lineares. Simulações numéricas mostram a eficiência do controle proposto.
\end{abstract}

Palavras-chave: Sistemas dinâmicos, controle ótimo, dengue.

\section{INTRODUÇÃO}

Epidemias incontroladas causadas por doenças infecciosas foram as principais causas de mortes no último milênio. A criação de vacinas, antibióticos e também as melhorias das condições de vida levou-nos a pensar que as doenças infecciosas desapareceriam, porém elas ainda continuam sendo a maior causa de mortalidade.

Uma das doenças infecciosas que pode causar a morte de humanos é a dengue. Ela é uma arbovirose que é transmitida para o homem através da picada do mosquito Aedes aegypti que, por ser domiciliado, é um transmissor eficiente da dengue. Os sorotipos da dengue são Den 1, Den 2, Den 3 e Den 4, sendo que um indivíduo infectado por um sorotipo se torna imune a esse sorotipo [1].

No Brasil, mortes causadas pela dengue continuam sendo notícia, apesar de serem realizadas campanhas de conscientização para diminuição de criadouros do mosquito e também a aplicação

\footnotetext{
*Autor correspondente: Alexandre Molter.

${ }^{1}$ Universidade Federal de Pelotas, Departamento de Matemática e Estatística, Campus Universitário, 354, 96010-900 Pelotas, RS, Brasil. E-mails: alexandre.molter@yahoo.com.br; rejane.pergher@gmail.com

${ }^{2}$ Universidade Federal de Pelotas, Centro de Engenharias, Av. Almirante Barroso, 1734, Centro, 96010-280 Pelotas, RS, Brasil. E-mail: lurpiovesan@gmail.com

${ }^{3}$ Universidade Federal do Rio Grande do Sul, Instituto de Matemática, Avenida Bento Gonçalves, 9500, Prédio 43-111, Bairro Agronomia, 91509-900 Porto Alegre, RS, Brasil. E-mail: cris@ mat.ufrgs.br
} 
de outros métodos para buscar um controle do mosquito e como consequência uma diminuição da doença. Por ser uma infecção viral urbana mais difundida, não apenas no Brasil mas em todo o mundo, várias pesquisas em modelagem de epidemias têm se preocupado em fornecer uma fundamentação racional para tomadas de decisão com o objetivo de controlar a propagação da doença.

Segundo [10] a erradicação do mosquito (vetor) através de mecanismos de controle tem sido uma estratégia adotada para prevenir as ocorrências de surtos de dengue, pois as vacinas contra a dengue ainda não estão disponíveis (encontram-se em fase experimental). Entre diversas estratégias de controle, podemos citar a utilizada por [2], que sugere e apresenta um modelo matemático para se controlar o mosquito transmissor da dengue, usando uma técnica de liberação de mosquitos machos estéreis. Em outros trabalhos, como em [3] e [8], é utilizado o controle ótimo para controlar o mosquito transmissor.

O objetivo deste trabalho é formular e aplicar uma estratégia de controle ótimo linear para sistemas não-lineares [5] que consistirá em reduzir os acumuladores de ovos dos mosquitos transmissores da Dengue e evitar a transmissão de humanos para mosquitos.

\section{O MODELO BÁSICO SEIR CONTÍNUO}

No modelo SEIR que estudaremos, [9] e [4], consideramos um habitat espacialmente homogêneo, isto é, que o comportamento das populações independa da posição que elas ocupam. Isso possibilita trabalhar com equações diferenciais ordinárias, sendo o tempo a única variável independente. Para habitats que apresentam heterogeneidade espacial, seria necessário considerar explicitamente, além da variável tempo, as variáveis que determinam a posição da população em cada instante de tempo, e assim, trabalharíamos com equações diferenciais parciais. Quanto à propagação da doença, nosso modelo baseia-se nas nas seguintes hipóteses: o vírus se propaga através de encontros entre mosquitos infecciosos e humanos suscetíveis, transformando estes últimos em humanos expostos (ou infectados), bem como através de encontros entre humanos infecciosos e mosquitos suscetíveis, transformando estes últimos em mosquitos expostos (ou infectados). Tanto os mosquitos quanto os humanos têm um período de incubação, durante o qual eles são classificados como expostos (infectados) mas ainda não são infecciosos, isto é, não transmitem a doença. Além disso, na escala de tempo considerada, os humanos infecciosos podem recuperar-se, tornando-se imunes, mas os mosquitos infecciosos não se recuperam, pois a expectativa de vida do mosquito é muito pequena quando comparada com a do vírus da dengue e, portanto, uma vez infecciosos, eles sempre poderão transmitir a doença durante o seu período de vida. Nesta escala de tempo, a população de humanos é considerada constante, pois a expectativa de vida dos humanos (dezenas de anos) é muito maior que a dos mosquitos (alguns dias).

Com base nestas hipóteses, a dinâmica populacional pode ser descrita através de um modelo compartimental com 8 equações diferenciais ordinárias, para as seguintes populações: $S(t), E(t)$, $I(t), R(t)$, de humanos suscetíveis, expostos, infecciosos e recuperados, respectivamente; e, para os mosquitos, a população $A(t)$, quando na forma aquática (ovos, larvas e pupas), além 
das populações de mosquitos fêmeas adultas $M_{1}(t), M_{2}(t), M_{3}(t)$, de suscetíveis, expostos e infecciosos, respectivamente. A população total de humanos, constante, será representada por $N$.

Iremos supor que todos os mosquitos fêmeas adultos contribuam para a oviposição. Para os humanos que, como citamos anteriormente, têm uma expectativa de vida muito maior que a dos mosquitos, não fazemos distinção de faixa etária, e assim supomos que todos possam procriar; humanos sempre nascem suscetíveis.

Definimos, a seguir, diversos parâmetros, cujas unidades representamos usando a notação [·], para unidade de $\cdot$, como segue:

- $\phi$ - a taxa per capita de oviposição dos mosquitos fêmeas; $[\phi]=$ ovos por fêmea por unidade de tempo;

- $k(0<k<1)$ - a fração de ovos viáveis que se transformarão em larvas, e $f(0<f<1)$, a fração destes, que são fêmeas; ambos são adimensionais; assim, o produto $k f \phi$ é a taxa de crescimento intrínseco per capita da população de mosquitos fêmeas na fase aquática;

- $C$ - a capacidade de suporte dos criadouros onde os mosquitos colocam seus ovos; $[C]=$ ovos;

- $\pi_{q}$ - taxa per capita de eclosão das pupas, para transformar-se em mosquito suscetível; $\left[\pi_{q}\right]=[t]^{-1}$

- $\mu_{q}$ - taxa per capita de mortalidade das larvas ou das pupas, na fase aquática; $\left[\mu_{q}\right]=$ $[t]^{-1}$; assim, $1 / \mu_{q}$ é a expectativa de vida das larvas ou pupas na fase aquática;

- $\mu_{f}$ - taxa per capita de mortalidade dos mosquitos adultos, em qualquer compartimento; $\left[\mu_{f}\right]=[t]^{-1}$; assim, $1 / \mu_{f}$ é a expectativa de vida dos mosquitos fêmeas adultos em qualquer compartimento;

- $\mu$ - taxa per capita de nascimentos dos humanos que é igual à taxa per capita de mortalidade dos humanos, em qualquer compartimento; $[\mu]=[t]^{-1}$;

- $\beta_{m}$ - frequência dos encontros entre humanos infecciosos e mosquitos suscetíveis, que são eficientes para a propagação do vírus, isto é, que transformam mosquitos suscetíveis em mosquitos expostos; $\left[\beta_{m}\right]=$ mosquitos expostos por encontro por unidade de tempo; o produto $\beta_{m} I$ é denominado força de infecção exercida pelos humanos;

- $\beta_{h}$ - frequência dos encontros entre mosquitos infecciosos e humanos suscetíveis, que são eficientes para a propagação do vírus, isto é, que transformam humanos suscetíveis em humanos expostos; $\left[\beta_{h}\right]=$ humanos expostos por encontro por unidade de tempo; o produto $\beta_{h} M_{3}$ é denominado força de infecção exercida pelos mosquitos;

- $\gamma$ - taxa per capita com a qual um mosquito exposto passa para infeccioso; $[\gamma]=[t]^{-1}$; assim, $1 / \gamma$ é o tempo médio de incubação dos mosquitos; 
- $\alpha$ - taxa per capita com a qual um humano exposto passa para infeccioso; $[\alpha]=[t]^{-1}$; assim, $1 / \alpha$ é o tempo médio de incubação dos humanos;

- $\eta$ - taxa per capita de recuperação dos humanos; $[\eta]=[t]^{-1}$; assim, $1 / \eta$ é o tempo médio de recuperação dos infecciosos, isto é, para adquirirem imunidade.

O sistema de equações diferenciais que modela a dinâmica é, portanto:

$$
\begin{aligned}
\frac{d A}{d t} & =k f \phi\left(M_{1}+M_{2}+M_{3}\right)\left(1-\frac{A}{C}\right)-\left(\pi_{q}+\mu_{q}\right) A \\
\frac{d M_{1}}{d t} & =\pi_{q} A-\left(\beta_{m} I+\mu_{f}\right) M_{1} \\
\frac{d M_{2}}{d t} & =\beta_{m} I M_{1}-\left(\gamma+\mu_{f}\right) M_{2} \\
\frac{d M_{3}}{d t} & =\gamma M_{2}-\mu_{f} M_{3} \\
\frac{d S}{d t} & =\mu N-\left(\beta_{h} M_{3}+\mu\right) S \\
\frac{d E}{d t} & =\beta_{h} M_{3} S-(\alpha+\mu) E \\
\frac{d I}{d t} & =\alpha E-(\eta+\mu) I \\
\frac{d R}{d t} & =\eta I-\mu R
\end{aligned}
$$

A hipótese de que a população total de humanos se conserva, de acordo com $S(t)+E(t)+I(t)+$ $R(t)=N$, constante, é verificada ao somar as quatro últimas equações de (2.1).

\section{PONTOS DE EQUILÍBRIO E ESTABILIDADE}

Representando por

$$
\left(A^{*}, M_{1}^{*}, M_{2}^{*}, M_{3}^{*}, S^{*}, E^{*}, I^{*}, R^{*}\right),
$$

um equilíbrio do sistema (2.1), o qual é obtido igualando a zero todas as taxas de variação, obtemos três equilíbrios biologicamente viáveis, a saber:

1. O equilíbrio $S_{0}$, que é caracterizado pela ausência de mosquitos e a população humana toda suscetível.

$$
\left(A^{*}, M_{1}^{*}, M_{2}^{*}, M_{3}^{*}, S^{*}, E^{*}, I^{*}, R^{*}\right)=(0,0,0,0, N, 0,0,0) .
$$


2. O equilíbrio $S_{m}$, de coexistência entre as populações de humanos e de mosquitos, porém livre de doença, ou seja, além da fase aquática, as populações de mosquitos e humanos são constituídas apenas por suscetíveis.

$$
\begin{gathered}
\left(A^{*} ; M_{1}^{*} ; M_{2}^{*} ; M_{3}^{*} ; S^{*} ; E^{*} ; I^{*} ; R^{*}\right) \\
=\left(C\left(1-\frac{1}{Q_{0}}\right) ; \frac{\pi_{q}}{\mu_{f}} C\left(1-\frac{1}{Q_{0}}\right) ; 0 ; 0 ; N ; 0 ; 0 ; 0\right),
\end{gathered}
$$

desde que $Q_{0}>1$, sendo

$$
Q_{0} \equiv \frac{k f \phi}{\mu_{f}} \frac{\pi_{q}}{\left(\pi_{q}+\mu_{q}\right)}
$$

que pode ser interpretado como o número de descendência básico, pois o fator $\frac{k f \phi}{\mu_{f}}$ representa a probabilidade de que, durante o tempo de vida dos mosquitos fêmeas, ovos por estes colocados transformem-se em larvas de mosquitos fêmeas, e o fator $\frac{\pi_{q}}{\left(\pi_{q}+\mu_{q}\right)}$ representa a probabilidade de que um indivíduo da fase aquática sobreviva transformando-se em um mosquito suscetível. Este número é adimensional.

3. O equilíbrio $S_{d}$, endêmico, onde

$$
\begin{aligned}
A^{*} & =C\left(1-\frac{1}{Q_{0}}\right) \\
M_{1}^{*} & =\frac{\left(\gamma+\mu_{f}\right) \mu_{f}(\alpha+\mu)(\eta+\mu)}{N \beta_{h} \beta_{m} \alpha \gamma\left[1-\frac{(\alpha+\mu)(\eta+\mu)}{\alpha \mu N} I^{*}\right]} \\
M_{2}^{*} & =\frac{\mu_{f}(\alpha+\mu)(\eta+\mu) I^{*}}{N \beta_{h} \alpha \gamma\left[1-\frac{(\alpha+\mu)(\eta+\mu)}{\alpha \mu N} I^{*}\right]} \\
M_{3}^{*} & =\frac{(\alpha+\mu)(\eta+\mu) I^{*}}{N \beta_{h} \alpha\left[1-\frac{(\alpha+\mu)(\eta+\mu)}{\alpha \mu N} I^{*}\right]} \\
S^{*} & =N-\frac{(\alpha+\mu)(\eta+\mu)}{\alpha \mu} I^{*}, \\
E^{*} & =\frac{\eta+\mu}{\alpha} I^{*}, \\
I^{*} & =\frac{R_{0}-1}{\mu_{f}+R_{0} \frac{(\alpha+\mu)(\eta+\mu)}{\alpha N \mu}} \\
R^{*} & =N-S^{*}-E^{*}-I^{*},
\end{aligned}
$$

desde que $R_{0}>1$ e $Q_{0}>1$, sendo

$$
R_{0} \equiv \frac{\gamma}{\gamma+\mu_{f}} \frac{\bar{M} \beta_{h}}{\mu_{f}} \frac{\alpha}{\alpha+\mu} \frac{N \beta_{m}}{\eta+\mu},
$$


onde

$$
\bar{M}=\frac{\pi_{q}}{\mu_{f}} C\left(1-\frac{1}{Q_{0}}\right),
$$

é o número de mosquitos suscetíveis no equilíbrio $S_{m}$.

Podemos observar que $R_{0}$ é o número reprodutivo básico da doença, pois ao introduzirmos um mosquito infeccioso em um meio onde todos são suscetíveis, este mosquito pica um número médio de $\frac{\bar{M} \beta_{h}}{\mu_{f}}$ humanos suscetíveis durante o seu período infeccioso, que é igual a $\frac{1}{\mu_{f}}$. Estes humanos, agora expostos, sobrevivem ao período de incubação com probabilidade $\frac{\alpha}{\alpha+\mu}$, e então, quando infecciosos, são picados por mosquitos suscetíveis numa média de $\frac{N \beta_{m}}{\eta+\mu}$, durante seu período infeccioso. A probabilidade desses mosquitos expostos sobreviverem o período de incubação e se tornarem infecciosos é dada por $\frac{\gamma}{\gamma+\mu_{f}}$. Portanto, concluímos que, para termos um caso efetivo da transmissão do vírus da dengue, é necesssário que um mosquito infectado pique um humano suscetível, tornando o humano infeccioso (após passar pelo período de incubação), e este, por sua vez, seja picado por um mosquito suscetível, tornando o mosquito infeccioso (também após passar pelo período de incubação).

Ao analisar a estabilidade [4] de cada um dos equilíbrios do sistema, conclui-se que $S_{0}$ é assintoticamente linearmente estável se $Q_{0}<1 ; S_{m}$ é assintoticamente linearmente estável se $Q_{0}>1$ e $R_{0}<1 ; S_{d}$ é assintoticamente linearmente estável se $Q_{0}>1$ e $R_{0}>1$.

Atribuindo aos parâmetros, valores realísticos baseados em [9] e [10],

$$
\begin{aligned}
& k=0,8 ; \quad f=0,8 ; \quad \phi=0,9 ; \quad C=1000 ; \quad \pi_{q}=0,8 ; \quad \mu_{q}=0,3 ; \quad \beta_{m}=0,005 \\
& \mu_{f}=0,3 ; \quad \gamma=0,125 ; \quad \beta_{h}=0,006 ; \quad \mu=0,07 ; \quad \alpha=0,1 ; \quad \eta=0,25 ; \quad N=1000
\end{aligned}
$$

sendo dias a unidade de tempo, obtivemos os gráficos da solução do sistema (2.1) apresentados na Figura 1. Segundo [9] e [10], estes parâmetros constituem condições favoráveis para a proliferação dos mosquitos em uma temperatura ambiente em torno de $30^{\circ}$ Celsius. Adotamos como condições iniciais os valores

$$
\left[A(0), M_{1}(0), M_{2}(0), M_{3}(0), S(0), E(0), I(0), R(0)\right]=[270,980,10,4,1000,0,0,0]
$$

Observamos que, passados os transientes, as populações tendem a: $A^{*}=283 ; M_{1}^{*}=253$; $M_{2}^{*}=355 ; M_{3}^{*}=148 ; S^{*}=73 ; E^{*}=382 ; I^{*}=119 ; R^{*}=426$, que são exatamente os valores obtidos substituindo os parâmetros adotados nas equações (3.3). Além disso, substituídos em (3.2) e (3.4) confirmamos que ambos são maiores do que 1.

Os gráficos foram construídos utilizando a resolução numérica de equações diferenciais ordinárias do software Matlab, através do método de Runge-Kutta de quarta ordem.

\section{ESTRATÉGIAS DE CONTROLE}

Podemos observar, através da Figura 1, que, com os parâmetros utilizados e valores iniciais dados por: apenas 10 mosquitos infectados, apenas 4 mosquitos infecciosos, 980 mosquitos suscetíveis, 

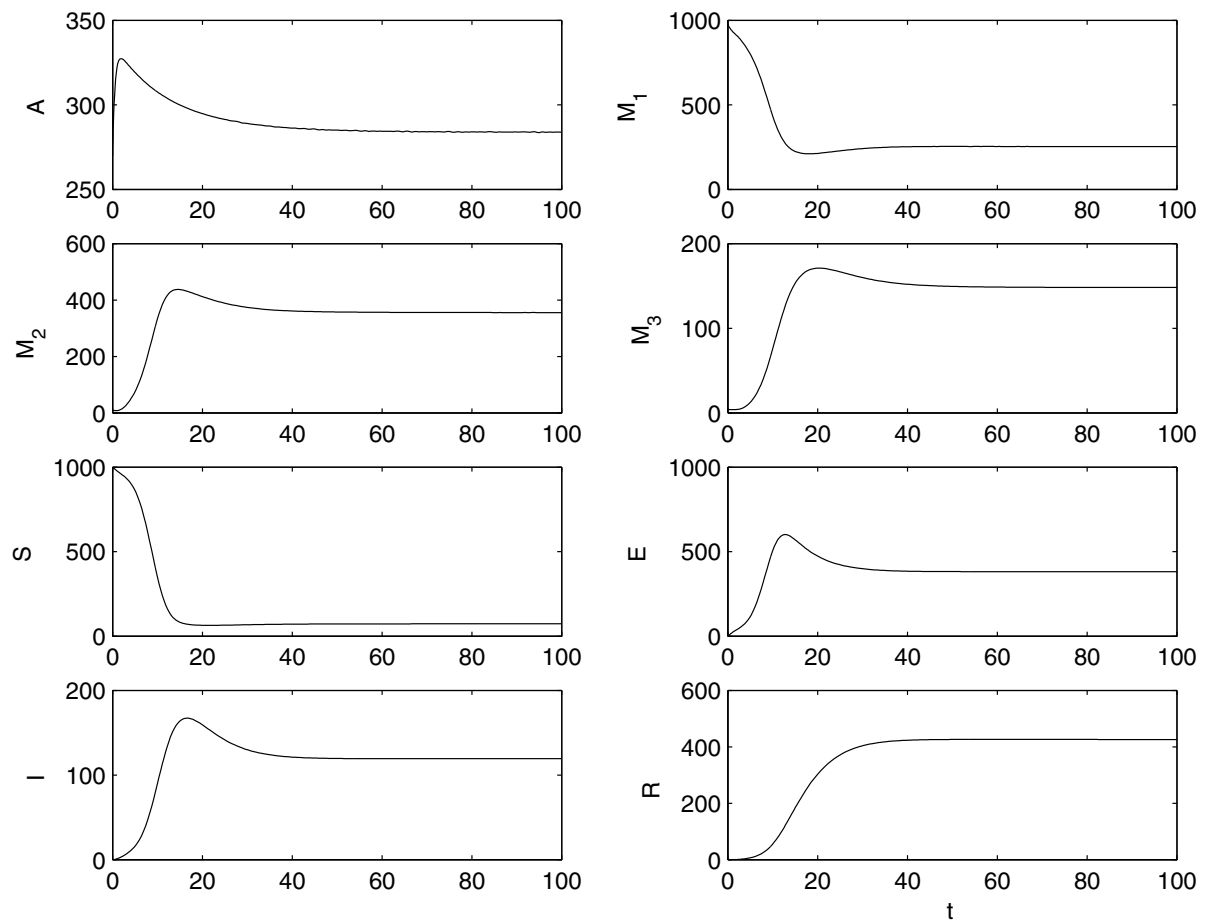

Figura 1: Gráficos da solução do sistema (2.1), correspondente aos parâmetros especificados na equação (3.6).

270 na fase aquática, juntamente com uma população de 1000 humanos todos suscetíveis, o sistema evoluiu para um equilíbrio endêmico, com grande propagação da doença em ambas as populações.

Com essa motivação, implementaremos nesta seção o uso de uma estratégia de controle de realimentação linear ótimo (optimal linear feedback control), apresentada em [7], aplicada à propagação da malária.

O objetivo deste controle é o de alterar o estado assintótico do sistema para $\left(\widetilde{A}, \widetilde{M}_{1}, \widetilde{M}_{2}, \widetilde{M_{3}}, \widetilde{S}\right.$, $\widetilde{E}, \widetilde{I}, \widetilde{R})$, de modo a manter a doença em níveis mais baixos.

Iremos supor que o controle da dengue se dá basicamente através do controle dos mosquitos. Neste trabalho o controle será efetuado mediante a redução dos mosquitos da dengue tanto na fase aquática $(A)$ quanto nos compartimentos de suscetíveis e infectados da fase adulta $\left(M_{1} \mathrm{e}\right.$ $M_{2}$ ). Para isso introduziremos variáveis de controle $U_{i}, i=1,2,3$, para as populações $A, M_{1}$ e $M_{2}$ respectivamente; essas variáveis de controle irão variar com o tempo, através das variáveis dependentes do sistema. A primeira corresponde à retirada de criadouros, que pode ser feito através da aplicação de inseticidas na água ou através da remoção de depósitos de água; enquanto que as duas últimas corrrespondem à aplicação de inseticidas no ar. 
Dessa forma trabalharemos com o seguinte sistema, obtido a partir de (2.1), introduzindo as variáveis de controle acima citadas:

$$
\begin{aligned}
\frac{d A}{d t} & =k f \phi\left(M_{1}+M_{2}+M_{3}\right)\left(1-\frac{A}{C}\right)-\left(\pi_{q}+\mu_{q}\right) A-U_{1}, \\
\frac{d M_{1}}{d t} & =\pi_{q} A-\left(\beta_{m} I+\mu_{f}\right) M_{1}-U_{2}, \\
\frac{d M_{2}}{d t} & =\beta_{m} I M_{1}-\left(\gamma+\mu_{f}\right) M_{2}-U_{3}, \\
\frac{d M_{3}}{d t} & =\gamma M_{2}-\mu_{f} M_{3}, \\
\frac{d S}{d t} & =\mu N-\left(\beta_{h} M_{3}+\mu\right) S, \\
\frac{d E}{d t} & =\beta_{h} M_{3} S-(\alpha+\mu) E, \\
\frac{d I}{d t} & =\alpha E-(\eta+\mu) I, \\
\frac{d R}{d t} & =\eta I-\mu R .
\end{aligned}
$$

A seguir consideraremos

$$
U_{i}=u_{i}+\tilde{u}_{i}, \quad i=1,2,3,
$$

de modo que o novo estado de equilíbrio, uma vez fixados os valores de $\widetilde{A}, \widetilde{M_{1}}$ e $\widetilde{M}_{2}$ desejados, deverá satisfazer o seguinte sistema algébrico com 8 equações e 8 incógnitas:

$$
\begin{aligned}
& k f \phi\left(\tilde{M}_{1}+\tilde{M}_{2}+\tilde{M}_{3}\right)\left(1-\frac{\tilde{A}}{C}\right)-\left(\pi_{q}+\mu_{q}\right) \tilde{A}-\tilde{u}_{1}=0, \\
& \pi_{q} \widetilde{A}-\left(\beta_{m} \widetilde{I}+\mu_{f}\right) \tilde{M}_{1}-\widetilde{u}_{2}=0, \\
& \beta_{m} \tilde{I} \tilde{M}_{1}-\left(\gamma+\mu_{f}\right) \tilde{M}_{2}-\tilde{u}_{3}=0 \text {, } \\
& \gamma \widetilde{M}_{2}-\mu_{f} \tilde{M}_{3}=0, \\
& \mu N-\left(\beta_{h} \tilde{M}_{3}+\mu\right) \tilde{S}=0, \\
& \beta_{h} \widetilde{M}_{3} \widetilde{S}-(\alpha+\mu) \widetilde{E}=0 \text {, } \\
& \alpha \widetilde{E}-(\eta+\mu) \widetilde{I}=0, \\
& \eta \widetilde{I}-\mu \widetilde{R}=0 \text {. }
\end{aligned}
$$

Com os desvios $u_{i}, i=1,2,3$, definidos acima, juntamente com os desvios $y_{j}, j=1, \ldots, 8$, definidos por:

$$
A=y_{1}+\widetilde{A}, M_{1}=y_{2}+\widetilde{M_{1}}, M_{2}=y_{3}+\widetilde{M}_{2}, M_{3}=y_{4}+\widetilde{M}_{3}
$$




$$
S=y_{5}+\widetilde{S}, E=y_{6}+\widetilde{E}, I=y_{7}+\widetilde{I}, R=y_{8}+\widetilde{R},
$$

obtém-se, a partir do sistema (4.1) juntamente com (4.3), o seguinte sistema de equações diferenciais na forma matricial:

$$
\dot{y}=\mathcal{A} y+\mathcal{H}(y)+\mathcal{B} \mathcal{U}
$$

onde $\mathcal{Y}(t)=\left[y_{1}(t) y_{2}(t) \ldots y_{8}(t)\right]^{T}$ e $\mathcal{U}=\left[u_{1} u_{2} u_{3}\right]^{T}$. As matrizes $\mathcal{A}, \mathcal{B}, \mathcal{H}(\mathcal{Y})$ são dados por:

$$
\begin{aligned}
& \mathcal{A}=\left(\begin{array}{cccccccc}
a_{11} & a_{12} & a_{13} & a_{14} & 0 & 0 & 0 & 0 \\
\pi_{q} & a_{22} & 0 & 0 & 0 & 0 & -\beta_{m} \widetilde{M}_{1} & 0 \\
0 & \beta_{m} \widetilde{I} & -\left(\gamma+\mu_{f}\right) & 0 & 0 & 0 & \beta_{m} \widetilde{M}_{1} & 0 \\
0 & 0 & \gamma & -\mu_{f} & 0 & 0 & 0 & 0 \\
0 & 0 & 0 & -\beta_{h} \widetilde{S} & a_{55} & 0 & 0 & 0 \\
0 & 0 & 0 & \beta_{h} \widetilde{S} & \beta_{h} \widetilde{M}_{3} & -(\alpha+\mu) & 0 & 0 \\
0 & 0 & 0 & 0 & 0 & \alpha & -(\eta+\mu) & 0 \\
0 & 0 & 0 & 0 & 0 & 0 & \eta & -\mu
\end{array}\right) \\
& a_{11}=-\frac{k f \phi}{C}\left(\widetilde{M}_{1}+\widetilde{M}_{2}+\widetilde{M}_{3}\right)-\left(\pi_{q}+\mu_{q}\right), \quad a_{12}=a_{13}=a_{14}=k f \phi\left(1-\frac{\widetilde{A}}{C}\right) \\
& a_{22}=-\left(\beta_{m} \tilde{I}+\mu_{f}\right), \quad a_{55}=-\left(\beta_{h} \widetilde{M}_{3}+\mu\right), \\
& \mathcal{H}(\mathcal{Y})=\left(\begin{array}{c}
-\frac{k f \phi}{C}\left(y_{1} y_{2}+y_{1} y_{3}+y_{1} y_{4}\right) \\
-\beta_{m} y_{7} y_{2} \\
\beta_{m} y_{7} y_{2} \\
0 \\
-\beta_{h} y_{4} y_{5} \\
\beta_{h} y_{4} y_{5} \\
0 \\
0
\end{array}\right) \\
& \mathcal{B}=\left(\begin{array}{ccc}
-1 & 0 & 0 \\
0 & -1 & 0 \\
0 & 0 & -1 \\
0 & 0 & 0 \\
0 & 0 & 0 \\
0 & 0 & 0 \\
0 & 0 & 0 \\
0 & 0 & 0
\end{array}\right)
\end{aligned}
$$


$\mathrm{Na}$ equação (4.5) para as derivadas dos desvios das populações, o primeiro e o segundo termos correspondem, respectivamente, aos termos lineares e quadráticos nos desvios destas populações, enquanto que o terceiro termo contém os desvios das variáveis de controle.

Para falar em controle ótimo, construímos o funcional custo que desejamos minimizar, como segue:

$$
J=\int_{0}^{T}\left(L(\mathcal{Y})+\mathcal{U}^{T} \mathcal{R} \mathcal{U}\right) d t
$$

onde $L(\mathcal{Y})=\mathcal{Y}^{T} \mathcal{Q} y-[\mathcal{H}(\mathcal{Y})]^{T} \mathcal{P} y-\mathcal{Y}^{T} \mathcal{P} \mathcal{H}(\mathcal{Y})$, envolve duas matrizes de ponderação $\mathcal{R}$ e $\mathcal{Q}$, onde $\mathcal{Q}$ é de ordem 8 e é relacionada ao custo social das populações. Os termos da matriz $\mathcal{R}$, quadrada de ordem 3 , são relativos aos custos dos inseticidas e das retiradas dos criadouros.

O teorema 4.1, retirado de [5], [6] e [7], apresenta condições suficientes para fazer uso de um controle de realimentação linear para sistemas não lineares e estabelece a forma do funcional otimizado.

Teorema 4.1. Se existirem matrizes $\mathcal{Q}(t)$ e $\mathcal{R}(t)$, positiva definidas, sendo $\mathcal{Q}$ simétrica, tais que a função

$$
L(\mathcal{Y})=\mathfrak{y}^{T} \mathcal{Q} \mathcal{Y}-[\mathcal{H}(\mathcal{Y})]^{T} \mathcal{P} y-\mathcal{Y}^{T} \mathcal{P} \mathcal{H}(\mathcal{Y})
$$

seja positiva definida, então o controle linear $\mathcal{U}$ dado por

$$
\mathcal{U}=-\mathcal{K} \mathcal{Y}
$$

onde $\mathcal{K}=\mathcal{R}^{-1} \mathcal{B}^{T} \mathcal{P}$, é ótimo para transferir o sistema não-linear (4.5) a partir de qualquer estado inicial ao estado final $\lim _{t \rightarrow \infty} \mathcal{Y}(t)=0$, minimizando o funcional

$$
J=\int_{0}^{\infty}\left(L(\mathcal{Y})+\mathcal{U}^{T} \mathcal{R} \mathcal{U}\right) d t
$$

onde a matriz simétrica positiva definida $\mathcal{P}(\forall t \geq 0)$ é a solução da formulação matricial da equação algébrica de Riccati

$$
\mathcal{P} \mathcal{A}+\mathcal{A}^{T} \mathcal{P}-\mathcal{P} \mathcal{B} \mathcal{R}^{-1} \mathcal{B}^{T} \mathcal{P}+\mathcal{Q}=0,
$$

que tem uma única solução positiva simétrica $\mathcal{P}>0$ para quaisquer $\mathcal{R}>0$ e $Q \geq 0$ dadas. Além disto, se tomarmos a função de Lyapunov $V=\mathcal{Y}^{T} \mathcal{P} Y$, verificamos que $\dot{V}$ é negativa definida, e consequentemente, o sistema controlado (4.5) tem estabilidade global.

\section{SIMULAÇÕES E RESULTADOS}

Inicialmente resolvemos o sistema (4.3) usando os mesmos parâmetros especificados na equação (3.6) e escolhendo para $\widetilde{A}, \widetilde{M}_{1}$ e $\widetilde{M}_{2}$ os valores 15,25 e 5 , respectivamente, obtendo o seguinte resultado:

$$
\begin{array}{ll}
\tilde{u}_{1}=1,7 ; \quad \tilde{u}_{2}=2 ; & \tilde{u}_{3}=0,3 ; \\
\tilde{M}_{3}=2 ; \quad \widetilde{S}=848 ; & \widetilde{E}=62 ; \quad \widetilde{I}=20 ; \quad \widetilde{R}=70 .
\end{array}
$$


A seguir, usamos a subrotina $L Q R$ do software Matlab, que utiliza como entrada as matrizes: $\mathcal{A}$, após substituídos os valores dos parâmetros e dos equilíbrios $\left(\widetilde{A}, \widetilde{M}_{1}, \widetilde{M}_{2}, \widetilde{M}_{3}, \widetilde{S}, \widetilde{E}, \widetilde{I}, \widetilde{R}\right)$ acima estabelecidos; $\mathcal{B}$ da equação (4.8); as matrizes de ponderação $\mathcal{Q}$ e $\mathcal{R}$ escolhidas como $\mathcal{Q}=\operatorname{diag}(1,1,110,1,1,1,1,15)$ e $\mathcal{R}=\operatorname{diag}(1,1,1)$. Como saídas, fornece as matrizes $\mathcal{P}$, que é a solução da equação (4.13) e a matriz $\mathcal{K}$, dada por:

$$
\left(\begin{array}{rrrrrrrr}
-0,5150 & -0,3286 & -0,0266 & 0,1060 & -0,0049 & 0,0182 & 0,0588 & 0,0249 \\
-0,3286 & -0,7979 & -0,1030 & -0,0383 & -0,0015 & 0,0101 & 0,0706 & 0,0081 \\
-0,0266 & -0,1030 & -10,2492 & -14,9577 & 0,2964 & -0,8311 & -1,4106 & -1,4517
\end{array}\right)
$$

com a qual construímos o controle linear (4.11).

Uma vez obtida essas matrizes e utilizando as mesmas condições iniciais com os quais construímos a Figura 1, implementamos o método de Runge-Kutta de quarta ordem para resolver o sistema (4.5). Para cada passo de tempo, calculamos um novo $\mathcal{U}$ a partir da equação (4.11) que após substituído na equação (4.5) fornece um novo sistema de equações diferenciais para os desvios, que será resolvido pelo método de Runge-Kutta, e assim por diante. Com essas soluções obtidas com o Matlab, para $t \in[0,100]$, os gráficos das soluções do sistema (4.1) são apresentados na Figura 2.
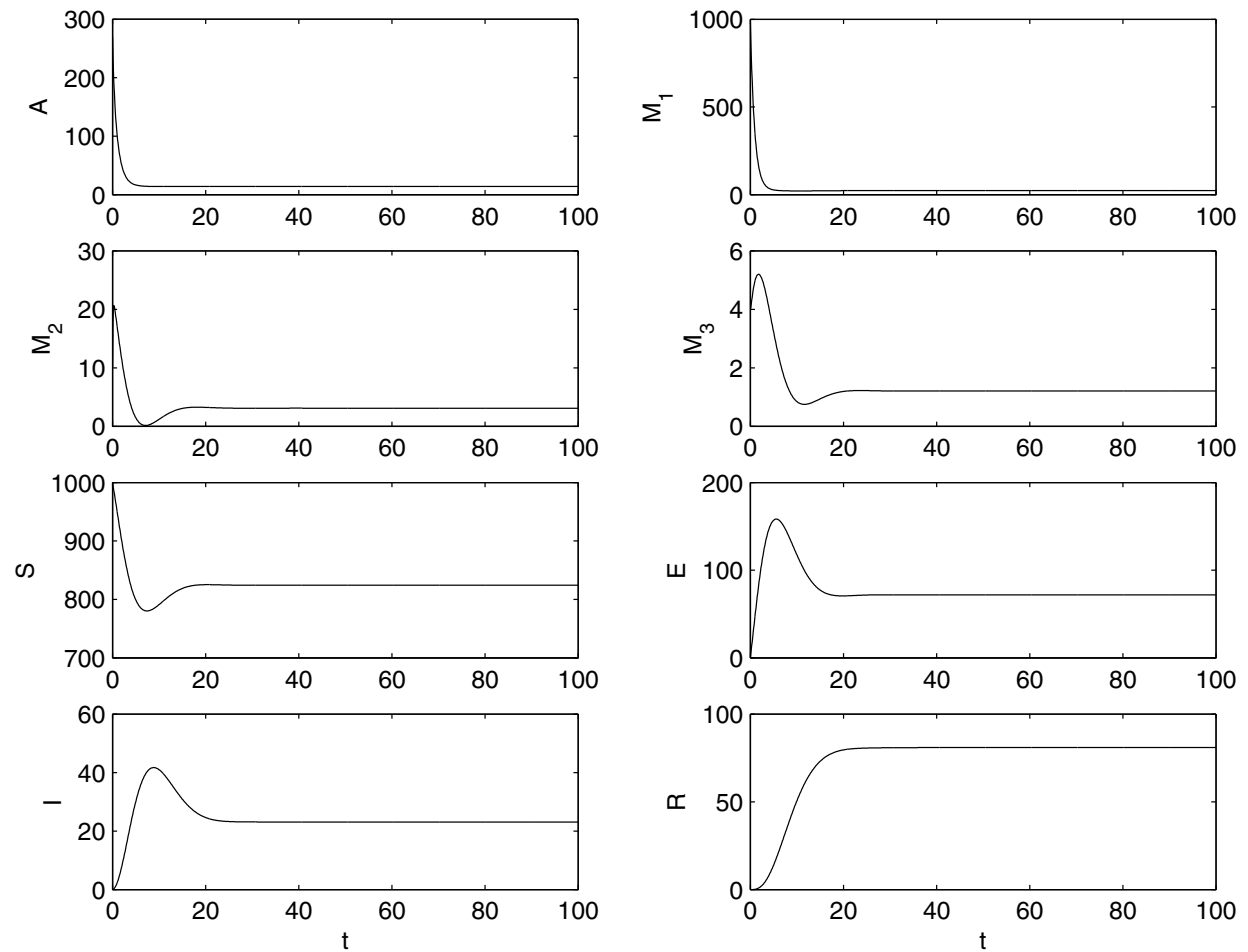

Figura 2: Gráficos da solução do sistema (4.1) com controle. 
Na Figura 2 podemos observar que após 20 dias o sistema atingiu um ponto de equilíbrio, próximo do desejado:

$$
\begin{aligned}
& \widetilde{A}=15 ; \quad \widetilde{M}_{1}=25 ; \quad \widetilde{M}_{2}=5 ; \quad \widetilde{M}_{3}=2 ; \\
& \widetilde{S}=848 ; \quad \widetilde{E}=62 ; \quad \widetilde{I}=20 ; \quad \widetilde{R}=70 .
\end{aligned}
$$

Em relação ao sistema sem controle, Figura 1, houve uma redução significativa das populações do agente causador da dengue, levando o nível de mosquitos infecciosos a menos de 2 indivíduos. Na população humana, cujo total é de 1000 indivíduos, aproximadamente 85\% mantém-se suscetível.

Para complementar, apresentamos na Figura 3, o gráfico do comportamento temporal da função $L(\mathcal{Y})$ da qual pelo teorema 4.1, equação (4.10), exigiu-se positividade para garantir convergência do sistema com controle (4.5).

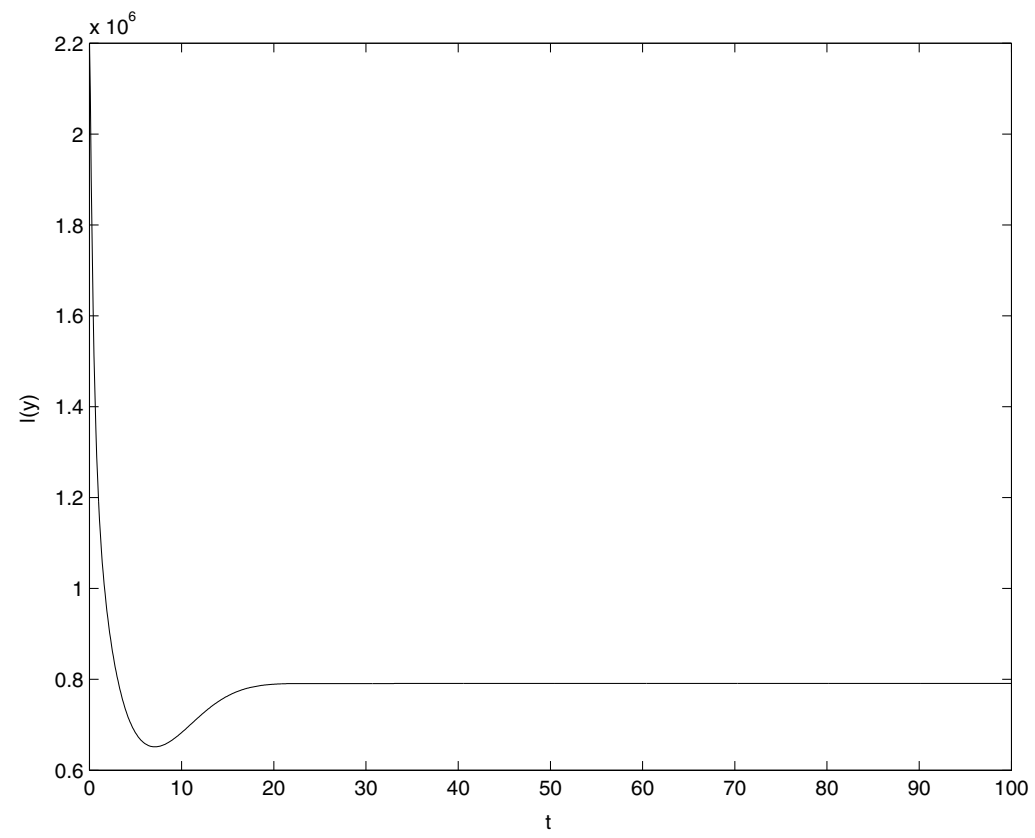

Figura 3: Evolução temporal da função positiva definida $L(\mathcal{Y})$ dada pela equação (4.10).

\section{CONCLUSÕES}

Neste trabalho foram apresentadas estratégias de controle em epidemias de dengue. O controle utilizado foi o controle ótimo linear para sistemas não-lineares. Mostrou-se que as estratégias de controle consideradas, através da retirada do mosquito da dengue do meio, foram eficientes. Embora o teor do trabalho seja teórico, as simulações mostraram eficiência com controle através da aplicação de inseticidas na água e no ar e a retirada de criadouros. Isto é positivo à medida que são a forma de combate mais utilizados atualmente para o controle da dengue. 
Com isso conclui-se que é possível reduzir as dimensões das epidemias, aprimorando o sistema de vigilância epidemiológica, detectando mais precocemente os surtos da doença e respondendo mais efetivamente no combate ao vetor. No entanto, isso implica em custos, tanto financeiros aos órgãos governamentais como custos sociais pela mobilização das comunidades.

Além de aprimorar as estratégias de controle apresentadas neste trabalho, há interesse em buscar outras estratégias como a liberação de mosquitos geneticamente modificados, que podem ampliar as possibilidades do controle da dengue.

\begin{abstract}
Dengue virus infection is nowadays one of the major worldwide public health problems. As there is no vaccine against dengue virus, it is important to have mathematical models that represent the dynamics of the epidemic aiming to control the spread of disease. In this work, we use a system SEIR continuous modeling the dynamics of dengue transmission and made a study of the stability of model with the biologically feasible equilibria points. The aim of this work is to apply alternative control strategies to reduce the spread of this infectious disease. The strategy used was the optimal control for non linear systems. Numerical simulations show the effectiveness of the proposed control.
\end{abstract}

Keywords: Dynamics systems, optimal control, dengue.

\title{
REFERÊNCIAS
}

[1] S. Cirino \& J.A.L. da Silva. Modelo Epidemiológico SEIR de Transmissão da dengue em redes de populações acopladas. TEMA - Tend. Mat. Apl. Comput., 5(1) (2004), 55-64.

[2] L. Esteva \& H.M. Yang. Control of dengue vector by the sterile insect technique considering logistic recruitment. TEMA - Tend. Mat. Apl. Comput., 7(2) (2006), 259-268.

[3] Q. Kong, Z. Qiu, Z. Sang \& Y. Zou. Optimal control of a vector-host epidemics model. Mathematical Control and Related Fields, 1(4) (2011), 493-508.

[4] R.P. Piovesan. "Modelo SEIR Discreto Espacialmente Estruturado para Dispersão da Dengue". Dissertação de Mestrado, Instituto de Matemática, Programa de Pós-Graduação em Matemática Aplicada, UFRGS, Porto Alegre, RS (2009).

[5] M. Rafikov \& J.M. Balthazar. On control and synchronization in chaotic and hyperchaotic systems via linear feedback control. Communications in Nonlinear Science and Numerical Simulation, 13 (2008), $1246-1255$.

[6] M. Rafikov, J.M. Balthazar \& H.F. von Bremen. Mathematical modeling and control of population systems: applications in biological pest control. Applied Mathematics and Computation, 200 (2008), $557-573$.

[7] M. Rafikov, L. Bevilacqua \& A.P.P. Wyse. Optimal control strategy of malaria vector using genetically modified mosquitoes. Journal of Theoretical Biology, 258 (2009), 418-425.

[8] H.S. Rodrigues, M.T.T. Monteiro \& D.F.M. Torres. Dynamics of dengue epidemics when using optimal control. Mathematical and Computer Modelling, 52 (2010), 1667-1673. 
[9] H.M. Yang, M.L.G. Macoris, K.C. Galvani, M.T.M. Andrighetti \& D.M.V. Wanderley. Assessing the effects of temperature on dengue transmission. Epidemiology and Infection, 137(08) (2009), 11791187.

[10] H.M. Yang \& C.P. Ferreira. Assessing the effects of vector control on dengue transmission. Applied Mathematics and Computation, 198 (2008), 401-413. 